\title{
Pharmacological characterisation of the antihyperglycaemic properties of Tinospora crispa extract
}

\begin{abstract}
The efficacy of Tinospora crispa (Menispermaceae) extract for the treatment of diabetes has previously been verified in animal models. In order to substantiate the antidiabetic effect, we characterised the antihyperglycaemic properties by studying its effect on intestinal glucose absorption and glucose uptake into adipocytes. We also performed experiments to characterise in more detail the mechanism of $\mathrm{T}$. crispa-evoked insulin release by challenging it with insulin secretory antagonists viz. adrenaline, somatostatin, verapamil and nifedipine. In addition, we also performed experiments to determine the effect of the extract on cAMP content. The results clearly showed that the antihyperglycaemic effect is not due to interference with intestinal glucose uptake or uptake of the sugar into the peripheral cells. Rather, the antihyperglycaemic effect of $\mathrm{T}$. crispa is probably due to stimulation of insulin release via modulation of $\mathrm{b}$-cell $\mathrm{Ca} 2+$ concentration. That the insulinotropic effect of $\mathrm{T}$. crispa is physiological suggests that the extract contains compounds which could be purified for use in the treatment of type II diabetes.
\end{abstract}

Keyword: Tinospora crispa; Diabetes; Antidiabetic; Medicinal plant; Insulin; Blood glucose 